\title{
Long-term clinical benefit of Peg-IFNa and NAs sequential antiviral therapy on HBV related HCC
}

\author{
Wen-Qian QI ${ }^{1, *}$, Qian ZHANG ${ }^{1, *, *}$, Xu WANG ${ }^{1}$, Yan XU1 ${ }^{1}$, Ping ZHAO ${ }^{1}$, Hong-Hua GUO ${ }^{1}$, Chang-Yu ZHOU ${ }^{1}$, Yu SUN ${ }^{2}$, Lin LIU², Jiang-Bin WANG ${ }^{1}$ \\ ${ }^{1}$ Department of Gastroenterology, China-Japan Union Hospital of Jilin University, Changchun, China; ${ }^{2}$ Department of Radiology, China-Japan \\ Union Hospital of Jilin University, Changchun, China \\ ${ }^{*}$ Correspondence: jiangbinwangdoc@126.com \\ ${ }^{*}$ Contributed equally to this work.
}

Received May 6, 2020 / Accepted July 21, 2020

\begin{abstract}
Analysis of the value of long-term antiviral therapy using sequential Peg-IFN therapy and nucleos(t)ide analogues (NAs) improves the prognosis of HBV-related HCC. HBV-related HCC patients were classified into sequential therapy with Peg-IFNa-2a and NAs, and NAs therapy alone. All patients were followed up for 5 years. The survival rate, HCC recurrence rate, Child-Pugh score, and side effects of drugs were evaluated. Firstly, the early and late cumulative survival rate was higher in patients receiving antiviral therapy compared with the control patients $(p<0.05)$. Patients receiving sequential therapy with Peg-IFNa-2a and NAs showed a higher late cumulative survival rate and significantly reduced early and late recurrence rate, compared to those in the NA-alone group $(\mathrm{p}<0.05)$. Single NAs therapy only reduced the late recurrence rate in HCC-patients. Secondly, NAs therapy significantly increased the Child-Pugh score after five years of therapy (fiveyear therapy $7.03 \pm 1.50$ vs. initial score $6.63 \pm 0.85 ; \mathrm{p}<0.05$ ), whereas the sequential therapy with Peg-IFNa-2a and NAs did not greatly alter the Child-Pugh score $(6.88 \pm 1.26$; $p>0.05)$. Compared to the control patients, patients receiving antiviral therapy (NAs alone or sequential therapy with Peg-IFNa-2a and NAs) exhibited a significantly decreased Child-Pugh score $(\mathrm{p}<0.05)$. Compared to NAs alone, sequential therapy with Peg-IFNa-2a and NAs provided a more efficient strategy for improving both the five-year survival rate and the two-year or five-year recurrence rate in patients.
\end{abstract}

Key words: primary hepatocellular carcinoma, Peg-IFN, nucleos(t)ide analogues, antiviral therapy

Primary hepatocellular carcinoma (HCC) is a common malignant tumor in the clinic, and over 626,000 cases are diagnosed annually worldwide. HCC has ranked the fifth most malignant tumor and the cancer-related mortality of HCC ranks the third most severe with approximately 600,000 deaths every year. In China, chronic liver disease is highly prevalent. HCC develops from chronic liver disorders, which account for $55 \%$ of the worldwide disease prevalence and is the second most common cancer-related mortality. A majority of Chinese HCC patients are affected by chronic $\mathrm{HBV}$ infection, which is different from that of western countries [1-3]. The annual prevalence of HCC is extremely high in liver cirrhosis patients with $\mathrm{HBV}$, and about one-third of these patients eventually develop HCC [4]. According to the BCLC guideline, curative resection, liver transplantation, or ablation is believed to be the most optimal strategy for the improvement of prognosis in Stage 0-1 HCC patients. However, HCC recurrence is still a major problem, which leads to poor prognosis [5-7]. It has been reported that the five-year recurrence rate in patients receiving liver resection or ablation can be as high as $50-85 \%$ [8].

The recurrence of HCC has been classified into early recurrence and late recurrence. Early recurrence, identified by HCC recurrence two years after curative resection or ablation, is predominantly associated with HCC metastasis [9]. Late recurrence, identified as HCC recurrence two years after therapy, mostly results from de novo tumors arising [10]. A recent study reveals that several factors contribute to $\mathrm{HCC}$ recurrence after curative resection, including tumor size, tumor mass number, portal vein invasion, AFP level, albumin level, and Child-Pugh score $[11,12]$. In addition, high HBV DNA load is known to be an independent risk factor for HCC recurrence [13-15] and is closely associated with late HCC recurrence $[16,17]$. In 2012, a nationwide cohort study in Taiwan showed that long-tern antiviral therapy using NAs significantly improved the survival rate in patients compared with control (89.4\% vs. 71.7\%) [18]. In addition, the six-year $\mathrm{HCC}$ recurrence rate in patients receiving antiviral therapy 
was greatly reduced compared to control (45.6\% vs. $54.6 \%)$ [18]. Shoji et al. [19] and Zimmerman et al. [20] reported that long-term antiviral therapy using LAM efficiently improved the long-term prognosis in HCC patients after curative resection and liver transplantation. The late recurrence of HCC patients receiving LAM antiviral therapy was reduced to $20 \%$ compared with a $70 \%$ five-year recurrence in control patients without antiviral therapy [19]. Nishikawa et al. [21] and Shin et al. [22] further confirmed the significance of NAs therapy such as LAM for improving the prognosis of HBV-related HCC.

A few studies have determined the association between IFN therapy and the recurrence of HBV-related HCC and shown that IFN therapy promoted the survival rate of patients by reducing early recurrence (one-year recurrence, $16.1 \%$ vs. $38.7 \%$ ) [10, 23, 24]. Compared to ordinary IFN, Peg-IFN has advantages in that it is stable and retains a high plasma drug concentration, as well as giving persistent antiviral responses (3-fold increase) [25-27]. Studies in patients with chronic HBV disorders demonstrated that sequential Peg-IFN therapy with NAs significantly elevated the persistent response rate $(36 \%$ vs. $14 \%$; $=0.011)$ and reduced drug resistance ( $21 \%$ vs. $40 \%$ ) compared with NAs therapy alone [28]. However, it remains unclear whether or not sequential Peg-IFN therapy with NAs promotes anti- tumor immune responses and reduces HCC recurrence in HBV-related HCC patients.

Based on this evidence, we investigated the potential improvement of long-term prognosis in HBV-related HCC patients after curative resection or ablation by long-term application of NAs antiviral therapy followed by Peg-IFN administration.

\section{Patients and methods}

Subjects. In this study, a total of 3108 primary HCC patients received curative resection or ablation in ChinaJapan Union Hospital, Jilin University from January 2003 to January 2013. HBV-related HCC patients with complete 5 -year follow up information $(n=256)$ were enrolled in the follow-up study. On the basis of patient willingness, patients were divided into two groups; 189 cases received antiviral therapy in the Digestive Disease Center, and 41 controls did not receive antiviral therapy. Among 189 patients receiving antiviral therapy, patients were further classified into sequential therapy with Peg-IFNa-2a and NAs $(n=40)$ and NAs therapy alone $(n=131)$. All patients were followed up for 5 years. The survival rate, HCC recurrence rate, Child-Pugh score, and side effects of drugs were evaluated (Figure 1). This study was conducted in accordance with the Declaration

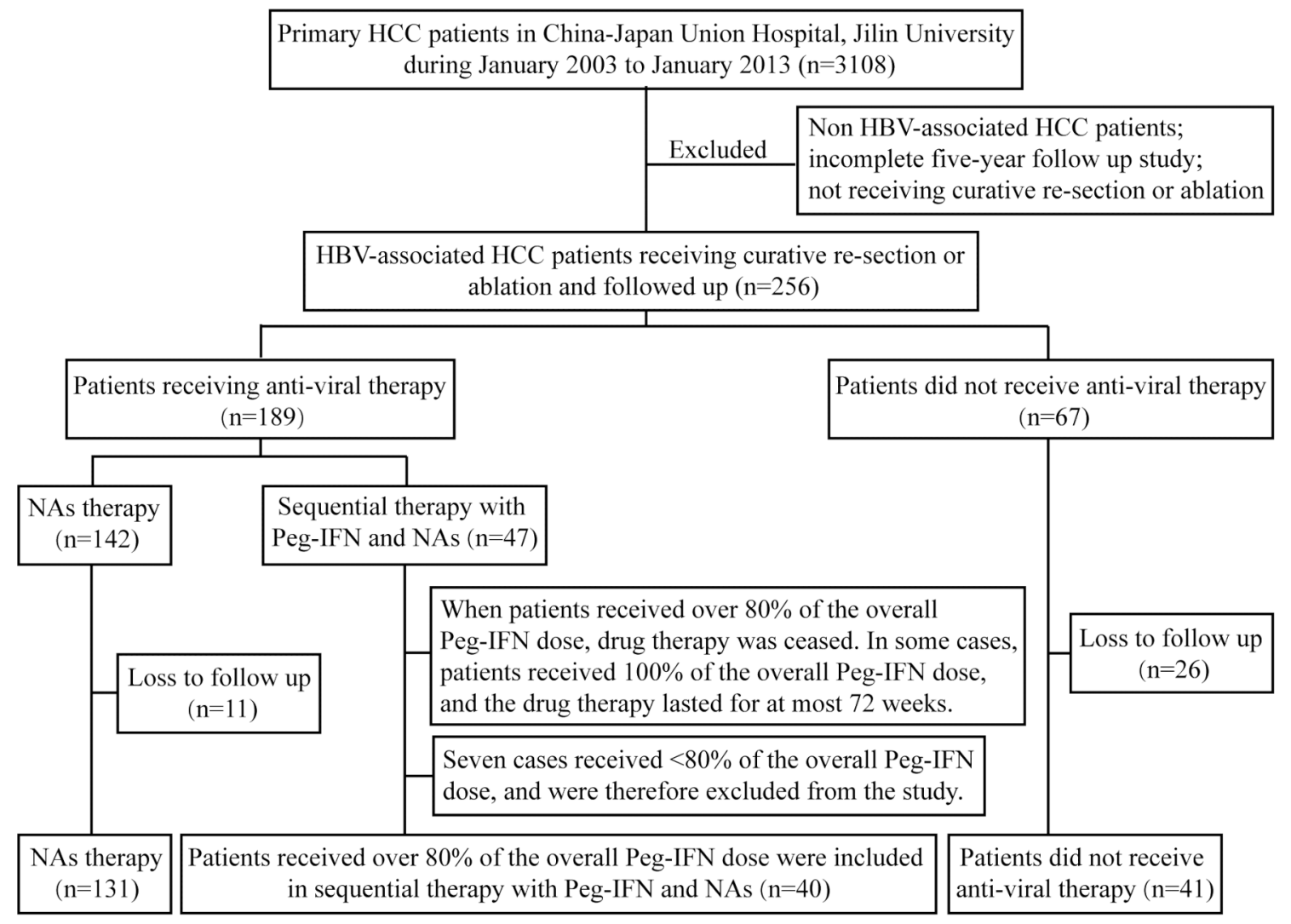

Figure 1. Flow diagram of the effects of antiviral therapy on the prognosis of HCC patients after curative resection or ablation. 
of Helsinki. This study was conducted with approval from the Ethics Committee of Jinlin University. Written informed consent was obtained from all participants.

Inclusion and exclusion criteria. Patients included in this study met the Clinical Practice Guidelines of HCC proposed by the NCCN in 2006 [29]. All patients with BCLC Stage 0-1 did not show HCC recurrence at three months following surgery. Inclusion criteria were as follows: 1) HBV DNA load $>1.0 \times 10^{3}$ copies $/ \mathrm{ml}$; 2) no chronic hepatitis $\mathrm{C}$ virus (HCV) infection or combined HIV infection determined by serological examination. Exclusion criteria were as follows: 1) patients had a history of antiviral therapy using NAs or Peg-IFNa; 2) patients received TACE therapy instead of curative resection or ablation; 3) HCC patients with residual tumor; 4) patients receiving systemic chemotherapy of Sorafenib; 5) patients with alcoholic liver disease, drug-induced liver disease, or autoimmune liver disease; 6) patients who died within three months after initial therapy.

Therapeutic strategy and experimental assignment. According to the antiviral therapy regimen, 212 patients after curative resection or ablation were classified into sequential therapy with Peg-IFNa-2a and NAs or NAs therapy alone. Patients receiving curative resection but without antiviral therapy were included as controls. All patients were followed up for 5 years.

Patients receiving sequential therapy with Peg-IFNa-2a and NAs were given Peg-IFNa-2a at an initial loading dose of $180 \mu \mathrm{g} / \mathrm{w}$. If the neutrophil count was $\leq 0.75 \times 10^{9} / 1$ or platelet count was $<50 \times 10^{9} / \mathrm{l}$, the dose of Peg-IFNa-2a was reduced to $135 \mu \mathrm{g} / \mathrm{w}$. In the case of patients being intolerant to therapy, the dose of Peg-IFNa-2a was reduced to 90 $\mu \mathrm{g} / \mathrm{w}$ or $67.5 \mu \mathrm{g} / \mathrm{w}$. Peg-IFNa-2a therapy was ceased when the neutrophil count was $\leq 0.5 \times 10^{9} / 1$ or platelet count was $<30 \times 10^{9} / 1$. When patients received over $80 \%$ of the overall Peg-IFN dose, drug therapy was ceased. In some cases,

Table 1. Demographic and clinical characteristics of 3108 patients with primary HCC.

\begin{tabular}{lc}
\hline Variable & Primary HC (n=3108) \\
\hline Gender (male:female) & $2285: 823$ \\
Age & $62.7 \pm 12.8$ \\
Etiology & \\
$\quad$ Chronic HBV infection & $2190(70.5 \%)$ \\
Chronic HCV infection & $431(13.9 \%)$ \\
Alcoholic fatty liver disease & $96(3.1 \%)$ \\
Others (including non-alcoholic fatty liver & \\
disease, autoimmune liver disease and & $391(12.5 \%)$ \\
unknown cause) & \\
Therapeutic approach & \\
Curative resection & $412(13.3 \%)$ \\
Ablation & $321(10.3 \%)$ \\
TACE & $1035(33.3 \%)$ \\
Others & $1340(43.1 \%)$ \\
\hline
\end{tabular}

patients received $100 \%$ of the overall Peg-IFN dose and the drug therapy lasted for a maximum of 72 weeks. Patients receiving $\geq 80 \%$ of the overall Peg-IFN dose were included in this study. Seven cases received $<80 \%$ of the overall Peg-IFN dose, and were therefore excluded from the study. After Peg-IFNa-2a withdrawal, patients were given $0.5 \mathrm{mg}$ ETV (q.d.) by oral administration.

Patients in the NAs therapy alone group were given $0.5 \mathrm{mg}$ ETV (q.d.) by oral administration. In cases where apparent drug resistance occurred, patients were given $0.5 \mathrm{mg}$ ETV (q.d.) plus $100 \mathrm{mg} \mathrm{ADV} \mathrm{(q.d.).}$

Measurements. One month after initial antiviral therapy, the HBV DNA load and liver function were analyzed. Thereafter, the HBV DNA load, liver function, renal function, AFP level, blood and routine urine outputs were evaluated once every three months. The imaging examination of the liver was conducted once every three months. The above indices were examined in control patients. For those with rebound HBV DNA, drug resistance was analyzed and the NA medications were adjusted according to the drug resistance sites.

Examinations: 1) Biochemical examination: liver function, renal function, blood and routine urine outputs were evaluated using an automatic biochemical analyzer and equipped reagents (Beckmann, USA). 2) HBV DNA: The serum HBV DNA load was assessed using the Roche Cobas PCR System. The lower limit of detection was 20 copies/ml. 3) HBV drug resistance: Serum HBV drug resistance mutations were examined by Sanger sequencing.

Statistical analysis. Data were analyzed by IBM SPSS 19 software (Chicago, IL, USA). Measurement data were expressed as $\chi \pm s$. The means were compared using the student's $\mathrm{t}$ test. Two-tail p-value $<0.05$ was considered statistically significant. $\chi^{2}$ test was used for comparing the rate. Cumulative prevalence and mortality rates were analyzed using the log-rank test.

\section{Results}

Demographic and clinical characteristics. A total of 212 HCC patients were enrolled in this study. Among these patients, 171 cases received antiviral therapy and 41 cases were included as controls. There was no significant difference in the gender, age, Child-Pugh classification, percentage of HBeAg-positive patients, AFP level, ALT level, HBV DNA loads, tumor mass number, tumor size, BCLC stage between control and HCC patients receiving antiviral therapy (Tables 1, 2).

Influences of sequential therapy with Peg-IFNa-2a and NAs on the cumulative survival rate. All patients included in the five-year follow-up study were evaluated for the influence of antiviral therapy on liver disease-related mortality. Although there was no significant difference in the cumulative survival rate at two years after initial therapy between patients receiving sequential therapy with Peg-IFNa-2a and NAs, and NAs alone, patients receiving sequential therapy 
Table 2. Comparison of the demographic and clinical characteristics between HCC patients receiving antiviral therapy and control.

\begin{tabular}{|c|c|c|c|c|}
\hline \multirow[b]{2}{*}{ Variable } & \multicolumn{2}{|c|}{ Antiviral therapy $(n=171)$} & \multirow{2}{*}{$\begin{array}{l}\text { Control } \\
(n=41)\end{array}$} & \multirow[b]{2}{*}{ p-value } \\
\hline & $\begin{array}{c}\text { Sequential therapy with } \\
\text { Peg-IFNa-2a and NAs }(n=40)\end{array}$ & $\begin{array}{l}\text { NAs therapy alone } \\
(n=131)\end{array}$ & & \\
\hline Gender (male/female) & $31 / 9$ & $96 / 35$ & $31 / 10$ & $>0.05$ \\
\hline Age (years, mean $\pm \mathrm{SD}$ ) & $51.8 \pm 5.8$ & $54.1 \pm 6.7$ & $55.3 \pm 7.0$ & $>0.05$ \\
\hline HBeAg (positive/negative) & $14 / 26$ & $44 / 87$ & $10 / 31$ & $>0.05$ \\
\hline ALT (IU/l, mean \pm SD) & $54.0 \pm 42.6$ & $54.7 \pm 64.3$ & $54.1 \pm 53.8$ & $>0.05$ \\
\hline $\mathrm{AFP}(\mathrm{ng} / \mathrm{ml}$, mean $\pm \mathrm{SD})$ & $239.4 \pm 246.8$ & $231.3 \pm 266.1$ & $229.3 \pm 304.2$ & $>0.05$ \\
\hline HBV DNA ( $\log _{10}$ copies/ml) & $4.8 \pm 1.0$ & $5.0 \pm 1.3$ & $5.0 \pm 1.1$ & $>0.05$ \\
\hline HBV subtype (type B/type C) & $13 / 27$ & $43 / 88$ & $13 / 28$ & $>0.05$ \\
\hline Liver cirrhosis & $35(87.5 \%)$ & $130(99.2 \%)$ & $37(90.2 \%)$ & $>0.05$ \\
\hline Child-Pugh stage (A/B) & $15 / 8$ & $52 / 78$ & $13 / 24$ & \\
\hline Tumor size $(\mathrm{cm}$, mean $\pm \mathrm{SD})$ & $2.4 \pm 0.5$ & $2.5 \pm 0.5$ & $2.6 \pm 0.4$ & $>0.05$ \\
\hline The number of tumor mass & 6 & 22 & 6 & $>0.05$ \\
\hline BCLC stage $(0 / 1)$ & $5 / 35$ & $22 / 109$ & $4 / 37$ & $>0.05$ \\
\hline Therapeutic approach & 14 & 40 & 13 & \\
\hline Curative resection (n) Ablation (n) & 26 & 91 & 28 & \\
\hline
\end{tabular}

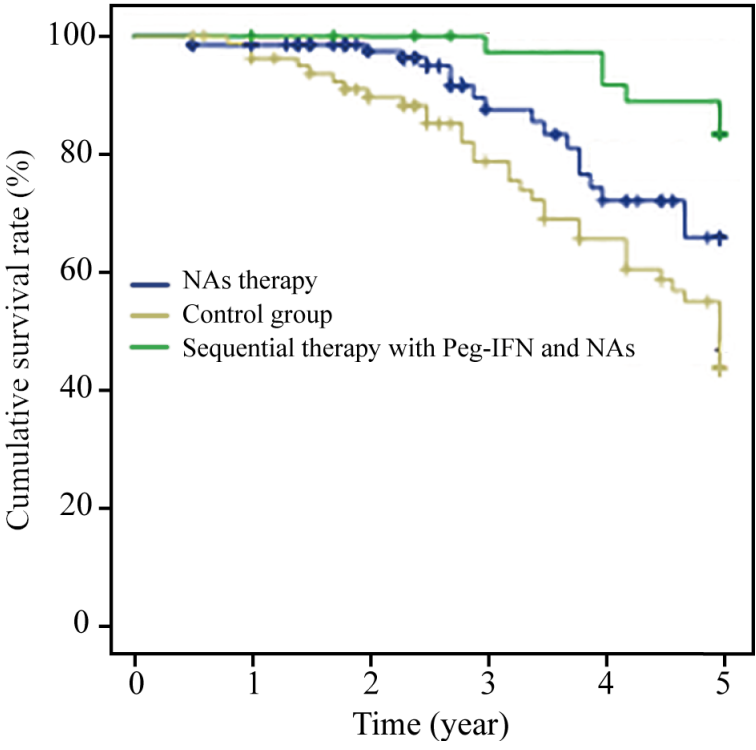

Figure 2. Influences of sequential therapy with Peg-IFN and NAs on the cumulative survival rate of HCC patients and the comparison between sequential therapy with Peg-IFN and NAs and NAs therapy alone. Twoyear cumulative survival rate: Sequential therapy with Peg-IFN and NAs vs. NAs, $p>0.05$; NAs vs. control, $p<0.05$. Five-year cumulative survival rate: Sequential therapy with Peg-IFN and NAs vs. NAs, $\mathrm{p}<0.05$; NAs vs. control, $\mathrm{p}<0.05$.

with Peg-IFNa-2a and NAs had a greatly increased cumulative survival rate at five years after initial therapy compared with those receiving NAs therapy alone $(\mathrm{p}<0.05)$. Compared to those receiving antiviral therapy, the early and late cumulative survival rate was remarkably reduced in patients without antiviral therapy $(\mathrm{p}<0.05$, Figure 2$)$.

Influences of sequential therapy with Peg-IFNa-2a and NAs on the cumulative recurrence rate. We compared the cumulative recurrence rate in patients receiving sequential

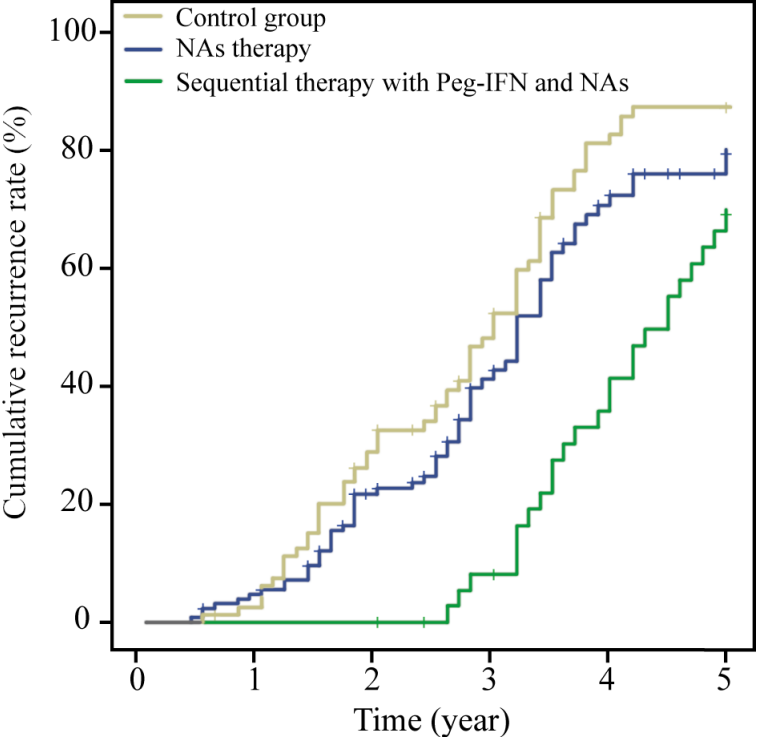

Figure 3. Influences of sequential therapy with Peg-IFN and NAs on the cumulative recurrence rate of $\mathrm{HCC}$ patients and the comparison between sequential therapy with Peg-IFN and NAs and NAs therapy alone. Twoyear cumulative recurrence rate: Sequential therapy with Peg-IFN and NAs vs. NAs, $p<0.05$; NAs vs. control, $p>0.05$. Five-year cumulative recurrence rate: Sequential therapy with Peg-IFN and NAs vs. NAs, $p<0.01$; NAs vs. control, $\mathrm{p}<0.05$.

therapy with Peg-IFNa-2a and NAs versus NAs alone. Our results showed that sequential therapy with Peg-IFNa-2a and NAs contributed to significantly reduced two-year and fiveyear recurrence rates compared with those receiving NAs alone $(\mathrm{p}<0.05)$. Although there was no significant difference in the two-year recurrence rate between the NAs group and the control group, patients receiving NAs therapy had a significantly lower five-year recurrence rate than control patients $(\mathrm{p}<0.05$, Figure 3$)$. 
Figure 4. Influences of antiviral therapy on the Child-Pugh score in HCC patients with liver cirrhosis.

Influences of antiviral therapy on the Child-Pugh score in HCC patients with liver cirrhosis. Among patients receiving antiviral therapy, 165 cases were identified as HCC combined liver cirrhosis, and 6 cases had no liver cirrhosis. The Child-Pugh score was not greatly altered in patients receiving sequential therapy with Peg-IFNa-2a and NAs during follow up study ( $p>0.05)$, whereas the ChildPugh score was gradually elevated in the NAs group, with time. In patients receiving NAs therapy alone, there was no significantly altered Child-Pugh score at two years following initial therapy compared with that before therapy $(\mathrm{p}>0.05)$. However, the Child-Pugh score at five years following initial therapy was significantly different $(7.03 \pm 1.50$ vs. $6.63 \pm 0.85 ; \mathrm{p}<0.05)$. Moreover, the Child-Pugh score at two years following initial therapy was significantly increased in patients without antiviral therapy when compared to those receiving antiviral therapy $(\mathrm{p}<0.05$, Figure 4$)$.

Analysis of drug resistance in patients receiving different antiviral therapy regimens. In patients receiving antiviral therapy, patients on sequential therapy with Peg-IFNa-2a and NAs were given Peg-IFN for one year followed by NAs therapy. After four years of antiviral therapy, none of the patients in this group developed drug resistance. However, in patients receiving NAs alone, two cases (4.1\%) developed ETV resistant mutations (at 2.5 years and 3 years after initial antiviral therapy). No new cases of drug resistance were identified 5 years after initial therapy. DNA sequencing showed that mutation occurred on the rtM204I/V, rtL180M, and rtT184 sites. For patients with drug resistance, ADV was administrated. Drug resistance was reversed after three months and six months of ETV plus ADV therapy in these two cases.

Analysis of side effects in patients receiving different antiviral therapy regimens. Side effects of antiviral therapy using Peg-IFNa-2a included fever, influenza-like syndrome, decreased neutrophil count in peripheral blood, reduced platelet counts, and alopecia. The most common side-effect of Peg-IFNa-2a therapy was fever, which occurred in $65 \%$ of patients receiving therapy. In addition, $57.5 \%$ of patients had a reduced white blood cell count, $47.5 \%$ of patients showed a reduced platelet count, and $45 \%$ of patients felt weak with myalgia. In some cases, blood glucose fluctuation $(12.5 \%)$, thyroid dysfunction (7.5\%), and clinical depression (5\%) were detected. No significant side-effects were noted in patients receiving NAs therapy alone.

\section{Discussion}

In China, chronic liver disease is highly prevalent. HCC develops from chronic liver disorders, which accounts for $55 \%$ of the worldwide disease prevalence and over $90 \%$ of Chinese HCC patients have chronic HBV infection [1-4]. According to the BCLC guideline, radical therapies, including curative resection, liver transplantation, or ablation, can be applied to Stage $0-1$ HCC patients, whereas the five-year survival rate of these patients is between $50-70 \%$ [30]. HCC recurrence is believed to be the major cause of poor prognosis [5-7].

Previous studies reveal that long-term antiviral therapy using NAs such as LAM efficiently reduced late recurrence rate and prolonged survival time in HCC patients after curative resection and liver transplantation [19-22, 31]. IFN not only has potent antiviral activity, but also regulates multiple biological events, including immune response regulation, anti-proliferation, and anti-angiogenesis. IFN is mainly used for the adjuvant therapy of HCV-associated HCC, and there are reports regarding the use of IFN for HBV-related HCC [32]. Single drug therapy may possibly lead to the rebound or recurrence of $\mathrm{HBV}$. Based on the experience of $\mathrm{HBV}$ management, sequential therapy with Peg-IFNa and NAs was used as adjuvant therapy for HBV-related HCC. Our results showed that, compared to NAs therapy alone, sequential therapy with Peg-IFNa-2a and NAs significantly improved the five-year survival rate $(83.3 \%)$ and reduced the two-year early recurrence rate as well as the five-year late recurrence rate. Yang et al. showed that adjuvant therapy using NAs provided a $73 \%$ (59-89.7\%) five-year overall survival in HBV-related HCC patients [33]. In their study, Asian HCC patients, especially in mainland China and Taiwan, received NAs adjuvant therapy after radiation excision. The characteristics of the cohorts were similar to that of our study and therefore has a certain significance for comparison. Our results demonstrated that sequential therapy with Peg-IFNa-2a and NAs significantly improved the five-year overall survival rate compared with NAs therapy alone, suggesting that sequential therapy with Peg-IFNa-2a and NAs has the ability to improve the longterm prognosis of HCC patients.

Most HBV-related HCC develops from liver cirrhosis. Despite sequential therapy with Peg-IFN and NAs being shown to improve the survival rate of patients, the impact of sequential therapy on the liver function of patients with 
liver cirrhosis is still unclear. Lv et al. showed that combined administration of LAM and ADV, or LAM alone gradually reduced the Child-Pugh score at 48, 96, or 144 weeks of therapy in patients with hepatitis B virus-related decompensated cirrhosis compared with that initial score before therapy (LAM combined ADV, 1.9, 2.6, 3.1; LAM alone, 1.7, 2.2, 2.7) [34]. Nevertheless, it remains unknown whether or not similar therapeutic outcomes can be achieved using antiviral therapy for $\mathrm{HBV}$-related HCC patients. In this study, we showed that there was no significant change in the Child-Pugh score in patients receiving sequential therapy with Peg-IFNa-2a and NAs during the follow-up study. However, the Child-Pugh score in patients receiving NAs therapy alone was increased at five years following initial therapy $(7.03 \pm 1.50$ vs. $6.63 \pm 0.85 ; \mathrm{p}<0.05)$. In addition, the Child-Pugh score at two years following initial therapy was significantly increased in patients without antiviral therapy when compared to those receiving antiviral therapy (either sequential therapy with Peg-IFNa-2a and NAs group, or NAs alone group). These data indicated that antiviral therapy could not improve the Child-Pugh score in HBV-related HCC patients. However, antiviral therapy efficiently delayed liver dysfunction. Moreover, sequential therapy with Peg-IFNa-2a and NAs appears to be more efficient in controlling disease progression when compared to NAs therapy alone.

Long-term administration of antiviral agents may lead to the risk of side effects and drug resistance. In this study, seven cases $(3.7 \%)$ were excluded from the study due to Peg-IFNinduced reduction of peripheral blood cells. These patients had to reduce the Peg-IFN dose or even withdraw from drug administration and received $<80 \%$ of the overall Peg-IFN dose. Side-effects of antiviral therapy using Peg-IFNa-2a included fever, fatigue, myalgia, decreased neutrophils in peripheral blood, reduced platelet counts, and alopecia. The most common side effect of Peg-IFNa-2a therapy was fever, which occurred in the majority of patients receiving therapy. A significant proportion of individuals experienced the following side-effects: reduced white blood cell count, reduced platelet count, fatigue, and muscular soreness compared to that of antiviral therapy against chronic HBV infection, the side-effects of Peg-IFNa-2a therapy are relatively increased [35]. However, all patients included were closely monitored and therefore no severe side-effects were found, suggesting the safety of sequential therapy with Peg-IFNa-2a and NAs for HCC patients. Drug resistance is known to be the major problem for antiviral therapy using NAs. In order to overcome NAs resistance $[36,37]$, ETV, which has a high genetic barrier of resistance, was used. Our findings indicated that two cases (1.5\%) developed drug resistance in the NAs therapy alone group, and none of the patients in the sequential therapy group showed drug resistance.

Considering China's healthcare system, we could not conduct a randomized controlled trial and therefore this prospective study was performed here. The follow-up duration is relatively long and some cases were lost to follow up in patients receiving antiviral therapy or not. This is both a strength and a weakness of the study.

In summary, sequential therapy with Peg-IFNa-2a and NAs had the advantages over NAs therapy alone in the efficient improvement of the long-term survival rate, decreased recurrence, and suppressed disease progression in HCC patients with liver cirrhosis after curative resection. At present, clinical studies focus on local therapy but fail to take into account the fact that antiviral therapy is of great importance as adjuvant therapy. Hence, the study of antiviral therapy for HBV-related HCC patients strengthens the significance of antiviral therapy for HBV-related HCC and may benefit the long-term prognosis and life quality in HCC patients.

Acknowledgments: This work was supported by the National Natural Science Foundation of China (No. 81670533), the Health and Family Planning Commission of Jilin Province (No. 2016Q016), and the Science \& Technology Department of Jilin Province (No. 20130102088JC).

\section{References}

[1] FASSIO E, DÍAZ S, SANTA C, REIG ME, MARTÍNEZ ARTOLA Y et al. Etiology of hepatocellular carcinoma in Latin America: a prospective, multicenter, international study. Ann Hepatol 2010; 9: 63-69.

[2] FENOGLIO L, SERRAINO C, CASTAGNA E, CARDELLICCHIO A, POMERO F et al. Epidemiology, clinical-treatment patterns and outcome in 256 hepatocellular carcinoma cases. World J Gastroenterol 2013; 19: 3207-3216. https:// doi.org/10.3748/wjg.v19.i21.3207

[3] LI M, LU C, CHENG J, ZHANG J, CAO C et al. Combination therapy with transarterial chemoembolization and interferon-alpha compared with transarterial chemoembolization alone for hepatitis B virus related unresectable hepatocellular carcinoma. J Gastroenterol Hepatol 2009; 24: 1437-1444. https://doi.org/10.1111/j.1440-1746.2009.05863.x

[4] SANGIOVANNI A, DEL NINNO E, FASANI P, DE FAZIO C, RONCHI G et al. Increased survival of cirrhotic patients with a hepatocellular carcinoma detected during surveillance. Gastroenterology 2004; 126: 1005-1014. https://doi. org/10.1053/j.gastro.2003.12.049

[5] SUN P, YANG X, HE RQ, HU QG, SONG ZF et al. Antiviral therapy after curative treatment of hepatitis $\mathrm{B} / \mathrm{C}$ virusrelated hepatocellular carcinoma: A systematic review of randomized trials. Hepatol Res 2014; 44: 259-269. https:// doi.org/10.1111/hepr.12115

[6] LlOVET JM, BURROUGHS A, BRUIX J. Hepatocellular carcinoma. Lancet 2003; 362: 1907-1917. https://doi. org/10.1016/S0140-6736(03)14964-1

[7] KUDO M. Adjuvant therapy after curative treatment for hepatocellular carcinoma. Oncology 2011; 81 Suppl 1: 50-55. https://doi.org/10.1159/000333259

[8] BRUIX J, SHERMAN M, AMERICAN ASSOCIATION FOR THE STUDY OF LIVER DISEASES. Management of hepatocellular carcinoma: an update. Hepatology 2011; 53: 1020-1022. https://doi.org/10.1002/hep.24199 
[9] MAZZAFERRO V, ROMITO R, SCHIAVO M, MARIANI L, CAMERINI T et al. Prevention of hepa- tocellular carcinoma recurrence with alpha-interferon after liver resection in HCV cirrhosis. Hepatology 2006; 44: 1543-1554. https:// doi.org/10.1002/hep.21415

[10] DU Y, SU T, DING Y, CAO G. Effects of antiviral therapy on the recurrence of hepatocellular carcinoma after curative resection or liver transplantation. Hepat Mon 2012; 12: e6031. https://doi.org/10.5812/hepatmon.6031

[11] KOIKE Y, SHIRATORI Y, SATO S, OBI S, TERATANI T et al. Risk factors for recurring hepatocellular carcinoma differ according to infected hepatitis virus-an analysis of 236 consecutive patients with a single lesion. Hepatology 2000; 32: 1216-1223. https://doi.org/10.1053/jhep.2000.20237

[12] SASAKI Y, YAMADA T, TANAKA H, OHIGASHI H, EGU$\mathrm{CHI} \mathrm{H}$ et al. Risk of recurrence in a long-term follow-up after surgery in 417 patients with hepatitis B- or hepatitis C-related hepatocellular carcinoma. Ann Surg 2006; 244: 771-780. https://doi.org/10.1097/01.sla.0000225126.56483.b3

[13] SHERMAN M. Risk of hepatocellular carcinoma in hepatitis $\mathrm{B}$ and prevention through treatment. Cleve Clin J Med 2009; 76 Suppl 3: S6-S9. https://doi.org/10.3949/ccjm.76.s3.02

[14] WU JC, HUANG YH, CHAU GY, SU CW, LAI CR et al. Risk factors for early and late recurrence in hepatitis B-related hepatocellular carcinoma. J Hepatol 2009; 51: 890-897. https:// doi.org/10.1016/j.jhep.2009.07.009

[15] SAAB S, YEGANEH M, NGUYEN K, DURAZO F, HAN S et al. Recurrence of hepatocellular carcinoma and hepatitis $B$ reinfection in hepatitis B surface antigen-positive patients after liver transplantation. Liver Transpl 2009; 15: 1525-1534. https://doi.org/10.1002/lt.21882

[16] PATERLINI P, POUSSIN K, KEW M, FRANCO D, BRECHOT C. Selective accumulation of the $\mathrm{X}$ transcript of hepatitis $\mathrm{B}$ virus in patients negative for hepatitis B surface antigen with hepatocellular carcinoma. Hepatology 1995; 21: 313-321.

[17] COLOMBO M, SANGIOVANNI A. Etiology, natural history and treatment of hepatocellular carcinoma. Antiviral Res 2003; 60: 145-150. https://doi.org/10.1016/j.antiviral.2003.08.010

[18] WU CY, CHEN YJ, HO HJ, HSU YC, KUO KN et al. Association between nucleoside analogues and risk of hepatitis $\mathrm{B}$ virus-related hepatocellular carcinoma recurrence following liver resection. JAMA 2012; 308: 1906-1914. https://doi. org/10.1001/2012.jama.11975

[19] KUBO S, TANAKA H, TAKEMURA S, YAMAMOTO S, HAI $S$ et al. Effects of lamivudine on outcome after liver resection for hepatocellular carcinoma in patients with active replication of hepatitis B virus. Hepatol Res 2007; 37: 94-100. https://doi.org/10.1111/j.1872-034X.2007.00013.x

[20] ZIMMERMAN MA, GHOBRIAL RM, TONG MJ, HIATT JR, CAMERON AM et al. Antiviral prophylaxis and recurrence of hepatocellular carcinoma following liver transplantation in patients with hepatitis B. Transplant Proc 2007; 39: 3276-3280. https://doi.org/10.1016/j.transproceed.2007.07.085
[21] NISHIKAWA H, NISHIJIMA N, ARIMOTO A, INUZUKA T, KITA R et al. Prognostic factors in patients with hepatitis B virus-related hepatocellular carcinoma undergoing nucleoside analog antiviral therapy. Oncol Lett 2013; 6: 1213-1218. https://doi.org/10.3892/ol.2013.1578

[22] SHIN HS, KIM SU, PARK JY, KIM DY, HAN KH et al. Antiviral efficacy of lamivudine versus entecavir in patients with hepatitis B virus-related advanced hepatocellular carcinoma. J Gastroenterol Hepatol 2012; 27: 1528-1534. https://doi. org/10.1111/j.1440-1746.2012.07145.x

[23] MIAO RY, ZHAO HT, YANG HY, MAO YL, LU X et al. Postoperative adjuvant antiviral therapy for hepatitis $\mathrm{B} / \mathrm{C}$ virusrelated hepatocellular carcinoma: a meta-analysis. World J Gastroenterol 2010; 16: 2931-2942. https://doi.org/10.3748/ wjg.v16.i23.2931

[24] XU JB, QI FZ, XU G, CHEN GF, HUANG MD et al. Adjuvant interferon therapy after surgical treatment for hepatitis B/C virus-related hepatocellular carcinoma: A meta-analysis. Hepatol Res 2014; 44: 209-217. https://doi.org/10.1111/ hepr. 12109

[25] COOKSLEY WG, PIRATVISUTH T, LEE SD, MAHACHAI $\mathrm{V}, \mathrm{CHAO}$ YC et al. Peginterferon alpha-2a $(40 \mathrm{kDa})$ : an advance in the treatment of hepatitis $\mathrm{B}$ e antigen-positive chronic hepatitis B. J Viral Hepat 2003; 10: 298-305. https:// doi.org/10.1046/j.1365-2893.2003.00450.x

[26] MARCELLIN P, ASSELAH T, BOYER N. Treatment of chronic hepatitis B. J Viral Hepat 2005; 12: 333-345. https:// doi.org/10.1111/j.1365-2893.2005.00599.x

[27] ZHU YY, WU YL, CHEN J, ZHENG Q, DONG J et al. [Prolonged duration of the routine pegylated-interferon alfa- $2 \mathrm{a}$ therapy produces superior virological response in $\mathrm{HBeAg}$ positive chronic hepatitis B patients: a single-center cohort study]. Zhonghua Gan Zang Bing Za Zhi 2012; 20: 737-741. https://doi.org/10.3760/cma.j.issn.1007-3418.2012.10.006

[28] ENOMOTO M, TAMORI A, NISHIGUCHI S, KAWADA N. Combination therapy with a nucleos $(\mathrm{t})$ ide analogue and interferon for chronic hepatitis B: simultaneous or sequential. J Gastroenterol 2013; 48: 999-1005. https://doi.org/10.1007/ s00535-012-0742-5

[29] BENSON AB 3RD, BEKAII-SAAB T, BEN-JOSEF E, BLUMGART L, CLARY BM et al. Hepatobiliary cancers. Clinical practice guidelines in oncology. J Natl Compr Canc Netw 2006; 4: 728-750. https://doi.org/10.6004/ jnccn.2006.0064

[30] LIN S, HOFFMANN K, SCHEMMER P. Treatment of Hepatocellular Carcinoma: A Systematic Review. Liver Cancer 2012; 1: 144-158. https://doi.org/10.1159/000343828

[31] SHEN, YC, HSU C, CHEN LT, CHENG CC, HU FC et al. Adjuvant interferon therapy after curative therapy for hepatocellular carcinoma (HCC): a meta-regression approach. J Hepatol 2010; 52: 889-894. https://doi.org/10.1016/j. jhep.2009.12.041

[32] ZHANG W, SONG TQ, ZHANG T, WU Q, KONG DL et al. Adjuvant interferon for early or late recurrence of hepatocellular carcinoma and mortality from hepatocellular carcinoma following curative treatment: A meta-analysis with comparison of different types of hepatitis. Mol Clin Oncol 2014; 2: 1125-1134. https://doi.org/10.3892/mco.2014.386 
[33] KE Y, WANG L, LI LQ, ZHONG JH. Nucleos(t)ide analogues to treat hepatitis B virus-related hepatocellular carcinoma after radical resection. World J Hepatol 2014; 6: 652-659. https://doi.org/10.4254/wjh.v6.i9.652

[34] LV GC, YAO JM, YANG YD, ZHENG L, SHENG JF et al. Efficacy of combined therapy in patients with hepatitis B virus-related decompensated cirrhosis. World J Gastroenterol 2013; 19: 3481-3486. https://doi.org/10.3748/wjg.v19. i22.3481
[35] CARUNTU FA, STREINU-CERCEL A, GHEORGHE LS, GRIGORESCU M, SPOREA I et al. Efficacy and safety of peginterferon alpha-2a (40KD) in HBeAg-positive chronic hepatitis B patients. Gastrointestin Liver Dis 2009; 18: 425431.

[36] LING R, MUTIMER D, AHMED M, BOXALL EH, ELIAS E et al. Selection of mutations in the hepatitis B virus polymerase during therapy of transplant recipients with lamivudine. Hepatology 1996; 24: 711-713. https://doi.org/10.1002/ hep.510240339

[37] LOK AS, MCMAHON BJ. Chronic hepatitis B. Hepatology 2007; 45: 507-539. https://doi.org/10.1002/hep.21513 